\title{
The epidemiology of human papillomavirus infection and cervical cancer
}

\author{
F. Xavier Bosch and Silvia de Sanjosé \\ Institut Català d'Oncologia, Epidemiology and Cancer Registration Unit, Av. Gran Via, s/n Km. 2,7, E-08907 \\ L'Hospitalet de Llobregat, Barcelona, Spain \\ Tel.: +34 93 2607812; Fax: +34 93 2607787; E-mail:x.bosch@ico.scs.es
}

\begin{abstract}
Cervical cancer has been recognized as a rare outcome of a common Sexually Transmitted Infection (STI). The etiologic association is restricted to a limited number of viral types of the family of the Human Papillomaviruses (HPVs). The association is causal in nature and under optimal testing systems, HPV DNA can be identified in all specimens of invasive cervical cancer. As a consequence, it has been claimed that HPV infection is a necessary cause of cervical cancer. The evidence is consistent worldwide and implies both the Squamous Cell Carcinomas (SCC), the adenocarcinomas and the vast majority (i.e. $>95 \%$ ) of the immediate precursors, namely High Grade Squamous Intraepithelial Lesions (HSIL)/Cervical Intraepithelial Neoplasia 3 (CIN3)/Carcinoma in situ. Co-factors that modify the risk among HPV DNA positive women include the use of oral contraceptives (OC) for five or more years, smoking, high parity (five or more full term pregnancies) and previous exposure to other sexually transmitted diseases such as Chlamydia Trachomatis (CT) and Herpes Simplex Virus type 2 (HSV-2). Women exposed to the Human Immunodeficiency Virus (HIV) are at high risk for HPV infection, HPV DNA persistency and progression of HPV lesions to cervical cancer.
\end{abstract}

Keywords: Cervical cancer, human papillomavirus, HPV, epidemiology

\section{Epidemiology of human papillomavirus infections and cervical cancer}

HPV infections of the cervix and the epidemiology of cervical cancer in relation to HPV are being investigated using as markers of exposure the presence of viral DNA in cellular scrapes or in biopsy tissue. Early DNA detection methods lead to the hypothesis that there was an association between cervical cancer and HPV exposure, however the development of Polymerase Chain Reaction (PCR) and other amplification techniques allowed an accurate assessment of the presence of viral DNA in cervical specimens as well as a quantification of the strength of the association down to the level of HPV type and of variants of the main types. Serological tests to detect antibodies to HPV antigens following natural infections are still in evaluation phase for large epidemiological studies. Natural HPV infections only trigger a measurable antibody response under special situations, resulting in significant under-detection.
In contrast antibody response to current HPV vaccines based on pseudo viral like particles (VLPs) from the L1 genomic region of type specific HPVs is universal and antibody titers are several fold (up to 40-fold) higher that antibody titers following natural infection. Therefore, most of the epidemiology of HPV and cervical cancer is largely based on DNA detection techniques of which most of the studies included in this review have used either the clinically validated Hybrid Capture 2 method (HC2) or some of the internationally validated PCR-based systems [1].

\subsection{Prevalence of human papillomavirus DNA. The international perspective}

The prevalence of HPV DNA in cervical specimens from the general population and from samples of women with normal cytology has been reported. The variability of results is considerable and relates to the selection of women included in the surveys (i.e. proba- 
bilistic samples of the population, women in screening programs and opportunistic series from clinical settings) and to the testing systems employed. Two recent sources have provided estimates that may reflect the global prevalence, the age-specific prevalence and the type-specific prevalence as well as an approximation to describing the international variability. In a multicentric and centrally coordinated international study, the International Agency for Research on Cancer (IARC) provided data from 15 areas in four continents among women 15-74 year old. The point age standardized prevalence was 10.5 (95\%CI 9.9-11.0) and ranged from a low end (less than 5\%) in Mediterranean countries and in some countries in South East Asia to a high end (greater than 15\%) in several countries in Latin America and in a few populations in Africa) [2].

The second source is a comprehensive review of the literature that used standardized criteria for study inclusion and statistical analyses that adjusted the estimates for the variables were influential in the comparability of studies. In this review, the estimates of the population prevalence worldwide (all ages) among women with normal cytology was of $10.41 \%$ with a $95 \%$ CI 10.2-10.7. The age specific prevalence showed higher proportions among the young age groups, a decline in the young adults and a variable pattern afterwards. In most countries, notably in the Americas, the prevalence increased again in the post-menopausal age groups. In Europe, the same pattern in the $40+$ age group is maintained in most surveys whereas in other high prevalence countries in Asia the HPV DNA prevalence remained fairly constant across all age groups [de Sanjose et al. in press]. Figure 1 shows the summary of the age-specific HPV DNA prevalence in women with normal cytology in a model that compiled data from close to 140,000 women reported in 67 studies from Africa (7 studies), America (17 studies), Europe (27 studies) and Asia (16 studies). The reason for the second increase in the HPV detection rate in the old age groups is unclear. The consistency of the finding in many individual studies in countries with substantial variability in sexual behaviour would indicate that the observation translates biological changes related to menopause-hormonal and immunological status that would facilitate HPV DNA detection from exposures earlier in life that remained silent/undetectable for a number of years. However, individual cohort studies and sexual behaviour surveys also indicate that additional number of new sexual partners habits in middle age and above might partially explain the second peak of genuine incident infections [3].

The HPV type-specific prevalence of the five most common HPV types in women with normal cytology is show in Fig. 2. HPV 16 is identified in $2.25 \%$ of women $(25.5 \%$ of positives) followed by HPV $18(0.8 \%)$, $31(0.8 \%), 58(0.7 \%)$ and $52(0.6 \%)$. This distribution reflects the biological advantage of HPVs 16 and 18 for persistency. The $33 \%$ relative proportion afforded by HPVs 16 and 18 combined in women with normal cytology is in contrast with the $70 \%$ relative frequency of these two viral types observed in invasive cervical cancer and translates the biological advantage for progression of HPV 16 and 18 also observed in several natural history studies [4].

\subsection{Behavioural determinants of human papillomavirus infection}

Several groups of studies have clearly shown that HPV is predominantly and largely transmitted through sexual intercourse. Other forms of transmission have been occasionally described, but its implication in cervical cancer is likely to be marginal, if any.

Epidemiological studies investigating risk factors for HPV infection clearly and consistently have shown that the key determinants among women are the number of sexual partners, the age at which sexual intercourse was initiated and the likelihood that at least one of her sexual partners was an HPV carrier as measured by his sexual behaviour traits [5]. The role of males as possible vectors of HPV was measured in the early epidemiological studies by questionnaires that addressed the sexual behaviour of the husbands or sexual partners of cervical cancer cases and controls. More recent studies had, in addition, the ability to measure HPV DNA in exfoliated cells from the penile shaft, the coronal sulcus and from the distal urethra [6].

Case-control studies have established that the risk of cervical cancer for a given woman is predictable by the sexual behaviour of her husband as much as for her own sexual behaviour. In populations where female monogamy is dominant, the population of female sex workers plays an important role in the maintenance and transmission of HPV infections. Moreover, the probability that a woman is an HPV carrier and her risk of developing cervical cancer have been shown to be related to the presence of HPV DNA in the penis or the urethra of her husband or sexual partner. These observations confirmed in terms of HPV infections a scientific hypothesis formulated almost 30 years ago that male sexual behaviour is a central determinant of the incidence of cervical cancer [7].

Studies on virgins offer a unique opportunity to demonstrate the predominantly sexual nature of HPV 


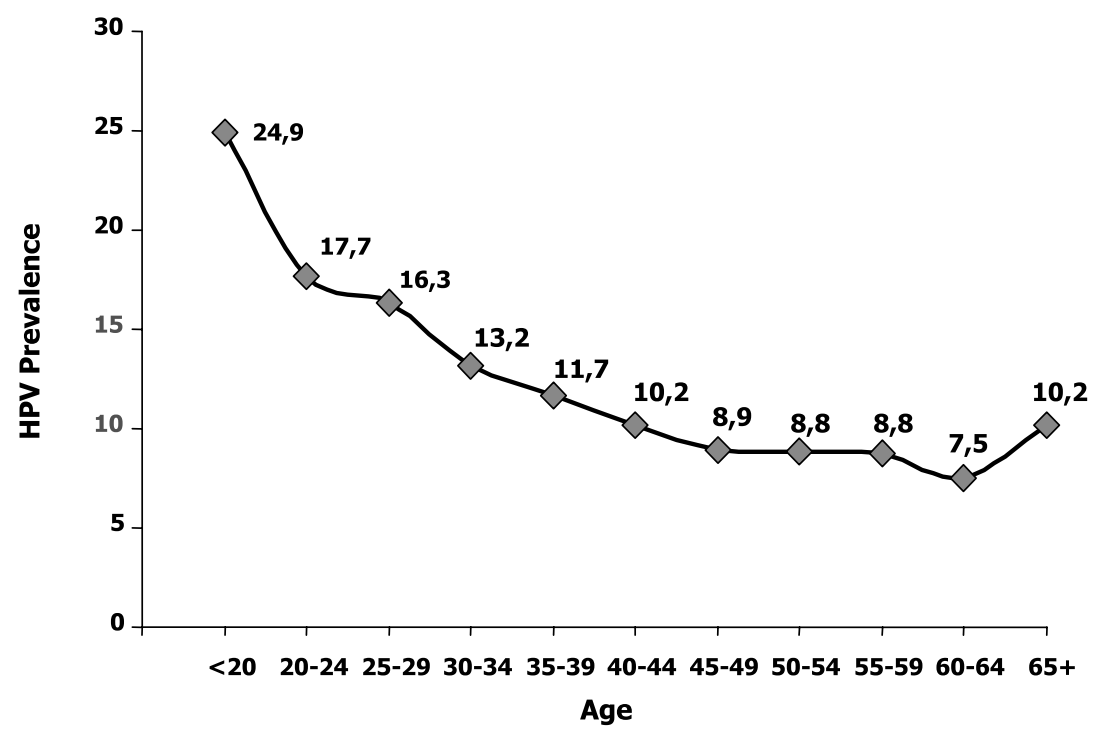

(De Sanjose et al in preparation)

Fig. 1. World-wide age-specific HPV-DNA prevalence among women from the general population.

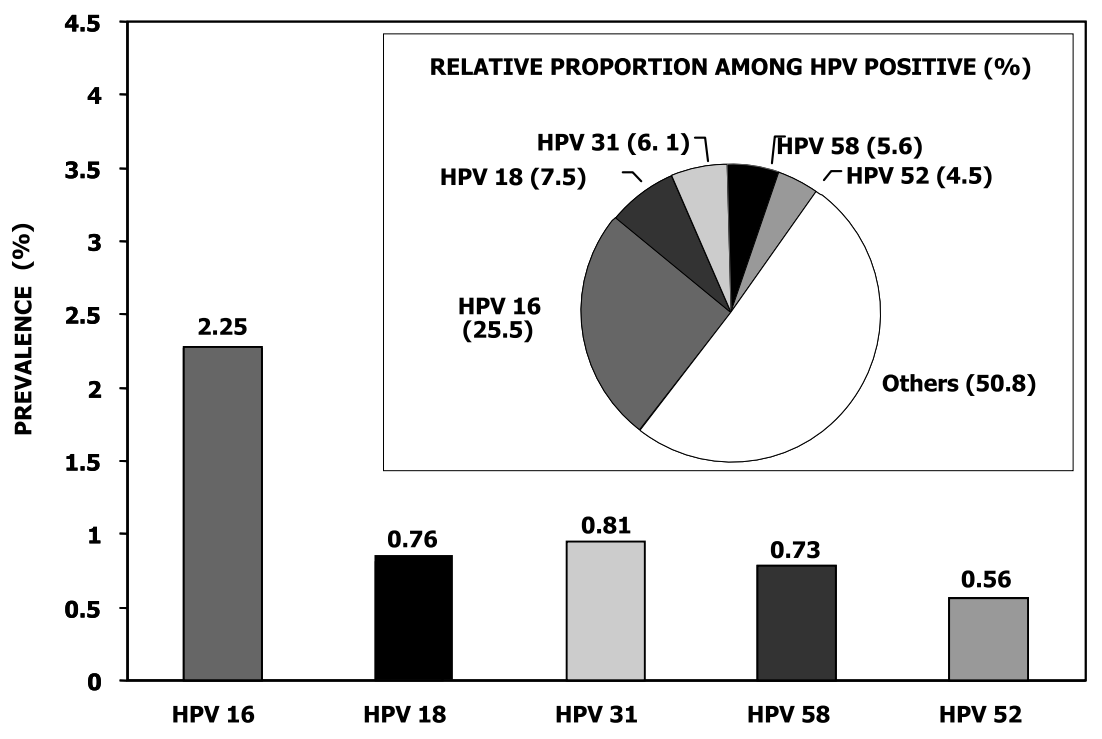

Source: Meta-analysis of 38 studies

HPV: Human Papillomavirus

Fig. 2. Overall HPV type specific prevalence \& relative proportion of the 5 most common types among HPV positive women with normal cytology.

transmission. Prevalence estimates of high risk HPV DNA infection from early studies in virgins range from $0 \%$ to $31 \%$. The wide range of these estimates indicates the influence of methodological issues including the sampling method used (scrapes vs. lavage), the subsite from which the samples were collected (cervical, vaginal or vulvar) and the HPV DNA detection method used. Variables of significant interest include the sensitivity of the test, the number of HPV types used in the testing system and the protocols followed to classify the HPV negative specimens and the HPV of unknown nature usually classed as HPV X. As HPV testing variability was better understood, several studies in which identical sampling methods were taken from all women 
in a closed cohort and in which HPV DNA was reliably measured, concluded that HPV DNA in cervical specimens is only detected in the sexually experienced women [5,8].

A longitudinal study of virgins who started sexual activity during the study period in a Danish populationbased cohort study of 100 virgins and 105 monogamous women showed that all women who stayed virginal throughout follow-up were consistently negative for both HPV DNA and HPV 16 serum antibodies at enrolment and at each follow-up visit. Only a fraction of those virgins who initiated sexual activity became positive for HPV DNA or HPV 16 serum antibodies. The most important determinant of HPV DNA acquisition in this study was the number of sexual partners the woman had had between enrolment and the follow-up visit, both among initially virginal women and among initially monogamous women [5]. In this study, detection of HPV 16 serum antibodies and development of cervical lesions occurred only after HPV transmission, suggesting that sexual intercourse is a necessary step in the acquisition of genital HPVs and in the development of the early stages of cervical neoplasia. A prospective study including 105 HPV-negative women carried out in the San Francisco bay area found that sexual behaviour, specifically exposure to new partners, represented the strongest risk factor for incident HPV infection. The association between sexual behaviour and incident HPV was quite notable, as the risk increased nearly 10-fold for each new partner per month reported.

Many epidemiological studies have consistently shown that sexual behaviour-related characteristics of the individual and his or her partners are the most important risk factors for the acquisition of genital HPV types. The relationship between number of partners and age at first intercourse with the corresponding detection of cervical and penile HPV DNA in adult women and men was examined among the controls $(2,225$ women and 1,140 men) of a series of 12 case-control studies of cervical cancer carried out by the IARC in 10 countries: Algeria , Brazil, Colombia, India, Morocco, Paraguay, Peru, Spain, Thailand and the Philippines. The study showed that, in both sexes, genital HPV DNA detection increased significantly with increasing lifetime number of sexual partners and with decreasing age at first sexual intercourse [7,8].

HPV data on social groups known to have or have had a high risk sexual behaviour, such as female sex workers and of attendees of STDs clinics have provided further evidence that HPVs are predominantly sexually transmitted. Two studies conducted in Spain and Den- mark compared HPV DNA prevalence among women from the general population and women belonging to high-risk groups In all age groups, HPV prevalence was highest among female sex workers, followed by women attending STDs clinics or incarcerated. Women from the general population had much lower age-specific HPV prevalence rates $[9,10]$.

\subsection{The role of male in the transmission of human papillomavirus and the risk of cervical cancer}

After the identification of HPVs as the sexually transmitted agents etiologically linked to cervical cancer, firm evidence for a role of men as carriers and vectors of oncogenic HPVs resulted from studies that introduced HPV DNA detection in penile samples. However, studies in males are limited because of lack of standardization of the methods to obtain cells from the male external genitalia. The largest study to date exploring the male role in cervical carcinogenesis using PCR technology for the detection of penile HPV is the multicentric case-control study coordinated by the IARC. This large study involved over 1,900 couples that were enrolled in one of seven case-control studies of cervical carcinoma in situ and cervical cancer carried out in Brazil, Colombia, The Philippines, Spain and Thailand [7,8,11]. Participating men answered a detailed risk-factor questionnaire and provided a specimen of exfoliated cells from the distal urethra, the glans and the coronal sulcus for HPV DNA detection.

Figure 3 shows the correlation between penile HPV DNA and the sexual behaviour of the couple for subjects enrolled in the studies conducted in Colombia [8] and Spain [7]. Penile HPV prevalence clearly increased with increasing numbers of sexual partners the men reported had had. The increase was observed in male partners of both monogamous and non-monogamous women and penile HPV prevalence was systematically higher in husbands of non-monogamous women than in husbands of monogamous women [6].

Findings from the IARC studies conducted in low- to intermediate-risk countries such as Spain, Thailand and the Philippines, indicate that the men's lifetime number of sexual partners and reporting sex workers as sexual partners are key determinants of cervical cancer risk in their wives. In Spain, the presence of HPV DNA in the husband's penis conveyed a 5-fold increased risk of cervical cancer to their wives. The odds of cervical cancer among monogamous women increased up to 9 to 10 fold in relation to the presence of high-risk HPV types in the penis of their husbands. The ex- 


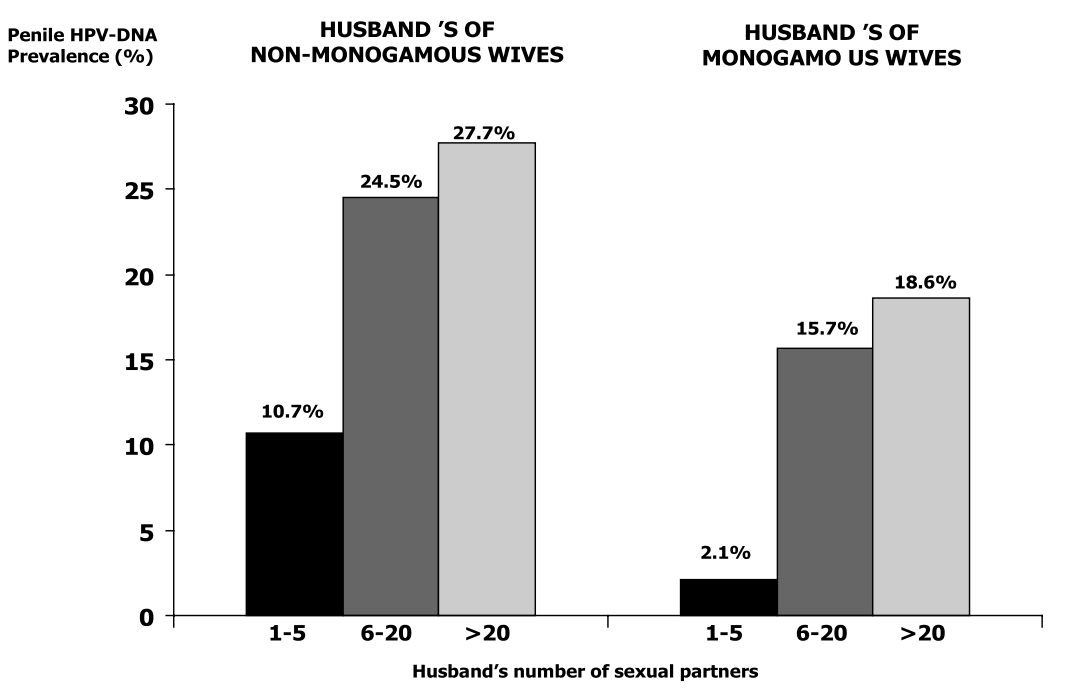

Source of data: Adapted from reference: IARC, 2005 [18]. Includes 595 men that were husbands or stable coital partners of women with and without cervical cancer.

Fig. 3. Penile Human Papillomavirus DNA prevalence by number of sexual partners of husbands of monogamous and non-monogamous women in Spain and Colombia.

SPAIN

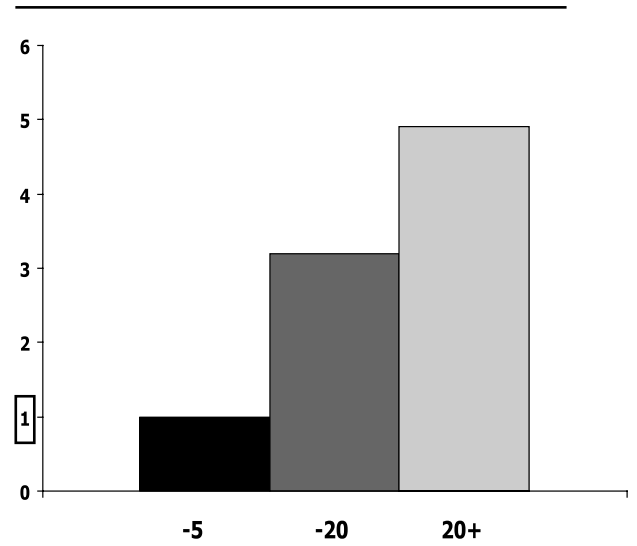

COLOMBIA

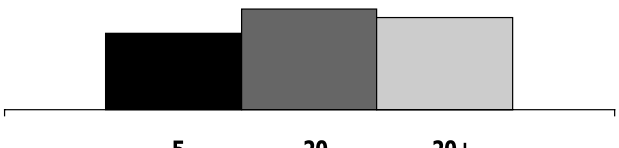

$-5$

Number of partners

Source of data: Adapted from reference: Muñoz N, et al., 1996 [8]

Fig. 4. Husband's lifetime number of partners and risk of cervical cancer among wives in a low risk (Spain) and a high risk country (Colombia).

cess risk associated with HPV type 16 was 6 to 9 fold. Furthermore, the prevalence of penile HPV showed a positive trend with increasing number of sexual partners and with the number of sexual partners who were sex workers [7]. In contrast, in high-risk countries such as Colombia and Brazil, no associations with cervical cancer risk were found with penile HPV DNA or with any other indicators of male sexual behaviour (Fig. 4) [8]. The lack of association between most male sexual behaviour-related variables and cervical cancer risk found in high-risk countries could be explained by the hypothesis that, in these populations, HPV is a widespread infection that it reduces the ability of case-control studies to discriminate subjects at a higher risk. Cross-sectional HPV DNA detection in the penis of adult men, even if high, is still a poor reflection of lifetime exposure to HPV. Other biological markers of lifetime sexual promiscuity in men such as seropositivity to Chlamydia Trachomatis (CT) are more consistent in discriminating women at a high-risk of cervical 
cancer in relation to the behaviour of their male sexual partners [12].

The clinical implications of recognizing the role of men as occasional carries of HPV DNA and of subclinical lesions that are source and routes of HPV transmission are still under evaluation. In some instances, careful examination of the male external genitalia of sexual partners of women with HPV DNA or CIN lesions unveils minute lesions amenable to treatment. These examinations, usually called penoscopy or genitoscopy, include observations under a colposcopic lens of the external genitalia including the scrotum and perianal regions, with or without acetic acid preparation. It is generally accepted that HPV visible lesions in men should be treated, however the lack of specific antiviral treatment detracts from recommendations of systematic examinations and additional diagnostic procedures and eventual treatments of male partners. Some literature is available indicating that the systematic and regular use of condoms accelerates regression of male and female lesions as well as of HPV DNA clearance [13].

Studies that have addressed concordance of genital HPVs in heterosexual couples found a relatively poor correlation of HPV positivity and HPV type in cervical and penile samples [6]. This is particularly important in the interpretation of case-control studies in which women harbours cervical neoplasia, thus a long-term consistent carrier of type-specific HPV-DNA whereas the husband is, or has been, a transient HPV DNA carrier. Moreover, in some couples, the current partner may not be the relevant one in determining the woman's risk of HPV persistence and progression to cervical neoplasia. Agreement in HPV findings, however, was also modest in couples where both - wife and husband reported only one lifetime sexual partner [14]. Among women with cervical neoplasia, the relevant infection may have occurred years earlier, and the relatively low prevalence of penile HPV infection in their husbands suggests that viral shedding of advanced cervical lesions is limited. Also cross-sectional detection of penile HPV may measure relatively recent exposures to HPVs that may be unrelated to the initiation of cervical neoplasia in the partner. Finally, the low agreement may be partly due to technical reasons, since a smaller amount of penile exfoliated cells may be obtained in men relative to the cellular yield obtained from the cervix.

The IARC multicentric study on male circumcision and its association with cervical cancer compared penile HPV DNA prevalence in circumcised and uncircumcised men and estimated the woman's risk of cer- vical cancer according to the husband's circumcision status. The study found that circumcised men were about 3 times less likely to harbour HPV in their penis than uncircumcised men. Male circumcision also reduced the risk of both genital HPV DNA prevalence and cervical cancer in the female partner, particularly and most strongly, in women whose male consorts had had a promiscuous sexual history [11]. Further, the protection afforded by male circumcision also refers to the seroprevalence of CT [15]. These findings, along with the literature on HIV transmission, also indicating a strong protection of circumcised men against HIV carriage and transmission, underline the relevance of circumcision for the prevention of STIs including HPV.

Transmission of genital warts, largely due to HPV 6 and 11 occurs during sexual intercourse with and infected partner. The infection is highly prevalent, with estimates of point prevalence around $1 \%$ the sexually active population with cumulative exposure of $17 \%$ in the age range 20 of $29 \%$ [16]. Risk factors have been clearly linked to the number of sexual partners and infectivity is high with transmission rates estimates of $60 \% 65 \%$ [17]. Genital warts do not convey high-risk of cervical cancer but HPV 6 and 11 have occasionally been related to rare forms of vulvar verrucous carcinomas of the Buschke-Lowestein type.

\subsection{Other routes of transmission of human papillomavirus. Recurrent respiratory papillomatosis}

Despite the overwhelming evidence that genital HPVs are predominantly sexually transmitted, some clinical and epidemiological observations have documented that genital HPV s can also be transmitted in other ways, especially from mother to child. This is consistent with other microbial and viral infections which are predominantly or exclusively sexually transmitted in adults (e.g., HIV, Hepatitis B Virus (HBV), HSV-2, CT, Treponema Pallidum (TP) and Neisseria Gonorrhoea (NG)) and that may be transmitted to newborns if present in the genital tract of a woman during pregnancy or at the time of delivery. The evidence for the non-sexual transmission of HPVs has been reviewed by several authors concluding that: 1) Genital HPV infections, including genital warts, may occur in sexually naïve populations such as virgins, infants, and children; 2) There is some evidence of horizontal transmission of low-risk HPVs; 3) Vertical and perinatal transmission of HPVs from mother to child, does exist, although rates are small and vary widely; 4) High-risk genital 
HPVs have been detected in non-genital mucosa, such as that in the mouth, oropharynx and conjunctiva, and they have been associated with a fraction of cancers of the oral cavity and oropharynx and with conjunctival SCC.

HPV transmission from the genital tract to the upper respiratory was first suggested in 1956, in a case report of a male child who was born to a mother with condyloma that developed symptoms of laryngeal papillomatosis and penile warts at 3 and 6 months after birth. Recurrent laryngeal papillomatosis is a rare, potentially life-threatening condition, associated with HPV types 6 and 11, the HPV types most commonly detected in genital warts. The disease can disseminate through the tracheobronchial tree and progress to pulmonary papillomatosis and subsequent fatal chest infection. Since the disease has a bimodal age distribution with the first peak occurring in infancy, it has been postulated that juvenile papillomatosis may be related to HPV infection acquired from a mother with genital warts or a subclinical HPV infection. A study showed that the risk of the juvenile form of laryngeal papillomatosis appeared to be highest in first-born infants delivered vaginally to an adolescent mother. In contrast, the risk factors identified for the adult onset of the disease included lifetime number of sex partners and high frequency of oral sex, suggesting orogenital transmission [18].

A number of other case report series have indicated the possible non-sexual horizontal transmission of HPVs, particularly of the low-risk HPV types. HPV DNA was detected in finger brush samples of 3 out of 8 women (one with the same types) with cervical HPV, and in 9 out of 13 men (5 with the same types) with penile HPV. In total, $27 \%$ of patients had the same HPV types detected in both genital and hand samples. These findings raise the possibility that patients with genital warts may transfer not only genital HPVs to their sexual partners by finger-genital contact but also horizontally to their children. Finger-conjunctiva HPV transmission has been suggested by studies reporting presence of HPV DNA, predominantly type 16, in human ocular surface squamous neoplasias, including conjunctival carcinomas. Indirect transmission via HPV-contaminated fomites (clothing, sheets, towels, objects, and instruments) has also been suggested by some studies, but its impact in passing and inducing active infections is most likely small if any [18]. Although HPV DNA has been occasionally isolated from medical instruments or in the event of medical examinations or treatments (i.e. at the time of laser ablation of CIN lesions in the vaporization fumes), there is at present no evidence that the isolated viral DNA is able to initiate an infection of the patient, her partner or the care taker.

\section{Human papillomavirus and cervical neoplastic lesions}

The evidence relating HPV infections to cervical cancer includes a large and consistent body of studies indicating a strong and specific role of the viral infection in all settings where investigations have taken place. The association has been recognized as causal in nature by a number of international review parties since the early 90's [19,20].

\subsection{Human papillomavirus DNA prevalence in cervical cancer specimens}

State-of-the art amplification techniques used in case-control studies, case-series and prevalence surveys have unequivocally shown that, in adequate specimens of cervical cancer, HPV DNA can be detected in 90 to $100 \%$ of the cases. Figure 5 shows the prevalence of HPV DNA in specimens of invasive cervical cancer identified in six regions of the world and investigated using PCR technology in a common centralized research laboratory. Allowing for the sampling and testing variability, these results are consistent with a universal presence of these viral markers in established cancerous lesions. The prevalence applies equally to the most common SCC and to the rarer adenocarcinoma, although the HPV type distribution in these two histological forms varies slightly. Further, detailed investigations of the few cervical cancer specimens that appear as HPV DNA negatives in most series has been occasionally conducted and the results strongly suggest that these are largely false negatives. As a consequence, the claim has been made that this is the first necessary cause of a human cancer ever identified, providing a strong rationale for the use of HPV tests in screening programs and for the development of HPV vaccines [21,22].

\subsection{Human papillomavirus types involved in cervical cancer and pre-cancerous lesions}

Of the more than 35 HPV types found in the genital tract, HPV 16 accounts for some $50 \%$ to $60 \%$ of the cervical cancer cases in most countries, followed by HPV 18: 10-20\%, HPV 45: 4-8\% and HPV 31: 


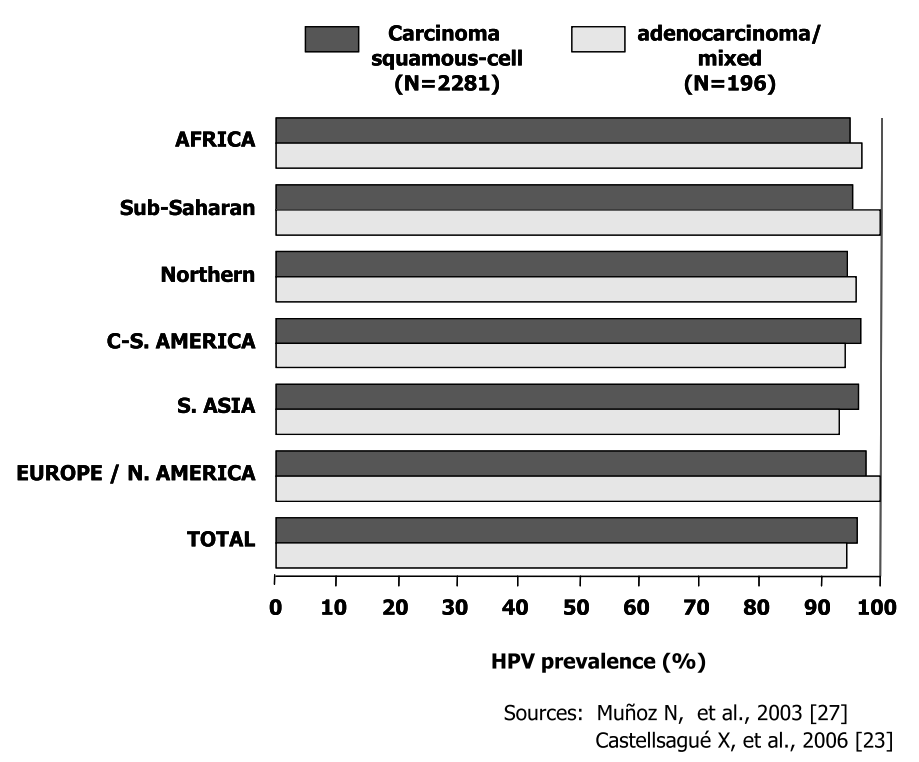

Fig. 5. HVP DNA prevalence in cervical cancer by study region and histology.

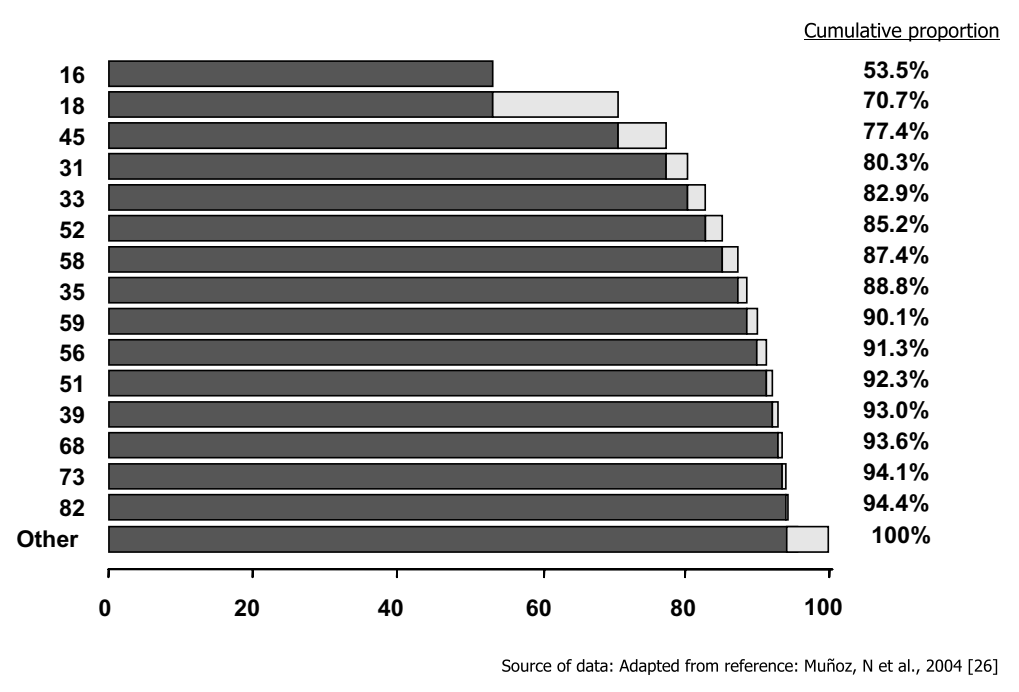

Fig. 6. Relative contribution of HPV types to cervical cancer: All world regions combined.

1-5\%. Figure 6 [23] shows the cumulative distribution of $15 \mathrm{HPV}$ types in a series of close to 3,000 cervical cancer cases. Of these, the 5 most common (HPVs 16, $18,45,31$ and 33 ) account for $80 \%$ of the distribution in squamous cell cancers and of $94 \%$ in adenocarcinomas. In most studies, HPV 18 predominates in adenocarcinomas in absolute or relative terms. The reasons for such specificity are unknown.

The positive selection of HPV 16 in cervical cancer cases across the cervical neoplastic spectrum translates the biological advantage of this type in fully expressing its oncogenic capacity. A study among wom- en with different degrees of HIV induced immunosuppression pointed at the likely increased ability of HPV 16 to escape immunesurveillance as compared to other HPV types as one possible mechanism of such advantage [24].

Table 1 compares the HPV type distribution in cervical cancer in the pre-neoplastic precursor lesions HSIL/CIN 2/3 and in women with normal cytology in the published meta-analyses of the literature. The table shows that HPVs 16, 18 and 45 are the only viral types that are more frequently found in the invasive form of neoplasia than in the precursor lesions. These 
Table 1

Comparison of overall and type-specific HPV prevalence between Squamous Cell Carcinoma (SCC), High Squamous Intraepithelial Lesion (HSIL) cases and women with normal cytology

\begin{tabular}{|c|c|c|c|c|c|c|}
\hline \multicolumn{7}{|c|}{ A summary of literature reviews } \\
\hline \multirow{2}{*}{$\begin{array}{l}\text { HPV } \\
\text { TYPE }\end{array}$} & \multicolumn{2}{|c|}{$\operatorname{SCC}(1)$} & \multicolumn{2}{|c|}{ HSIL (1) } & \multicolumn{2}{|c|}{ NORMAL CYTOLOGY (2) } \\
\hline & $\mathrm{n}$ & $\operatorname{HPV}(\%)$ & $\mathrm{n}$ & $\operatorname{HPV}(\%)$ & n women tested & $\mathrm{HPV}+\%$ \\
\hline ALL & 8550 & 87.6 & 4338 & 84.2 & 5.764 & 10.41 \\
\hline 16 & 8594 & 54.3 & 4338 & 45.0 & 4.385 & 2.25 \\
\hline 18 & 8502 & 12.6 & 4338 & 7.1 & 4.286 & 0.76 \\
\hline 33 & 8449 & 4.3 & 4302 & 7.2 & 4.136 & 0.52 \\
\hline 45 & 5174 & 4.2 & 2214 & 2.3 & 3.554 & 0.42 \\
\hline 31 & 7204 & 4.2 & 4036 & 8.8 & 4.157 & 0.81 \\
\hline 58 & 5646 & 3.0 & 2175 & 6.9 & 4.155 & 0.73 \\
\hline 52 & 5304 & 2.5 & 2153 & 5.2 & 3.927 & 0.56 \\
\hline $\begin{array}{l}\text { HPV: } \\
\text { SCC: } \\
\text { HSIL: } \\
\text { Source } \\
\text { Sanjos }\end{array}$ & $\begin{array}{l}\operatorname{man} \mathrm{P} \\
\text { lamou } \\
\text { gh Squ } \\
\text { of data } \\
\text { al. (i }\end{array}$ & $\begin{array}{l}\text { illomaviru } \\
\text { Cell Carcin } \\
\text { mous Intra } \\
\text { Adapted } \\
\text { press). }\end{array}$ & $\begin{array}{l}\text { elial } \\
\text { refer }\end{array}$ & $\begin{array}{l}\text { sion. } \\
\text { (1) G. }\end{array}$ & Cliffor & (2) $\mathrm{s}$ \\
\hline
\end{tabular}

findings are interpreted as if the ability to progress was increased in HSIL lesions due to these three HPV types than the corresponding lesions attributed to other HPV types [25]. These results are consistent with some observations from cohort studies indicating that the probability or progression given persistence is significantly higher in women exposed to HPVs 16 or 18 that to any other viral types [4].

The distribution of HPV types in cervical cancer in five different regions in the world is described in Table 2 [26]. It is clear that HPVs 16 and 18 are consistently the first two types involved in all regions explored so far. HPV 45 seems to be a natural candidate for the third ranking place and some additional variability exists thereafter for the relatively rarer HPV types.

\subsection{Risk estimates from case control studies}

Observations on pre-invasive disease and cohort studies on the HPV natural history have intrinsic limitations for making inferences on cervical cancer causality. This is because in controlled settings, biological progression is not allowed to continue beyond the stage of high-grade squamous intraepithelial lesions (HSIL/CIN 3) or carcinoma in situ. It is thus of importance to notice that the information applicable to cervical cancer would primarily come from case control studies in which the target disease is directly investigated.

In an effort to simplify the vast literature of case control studies, the results of the IARC multicenter case control study on invasive cervical cancer will be used as example. In brief, this project included nine case- control studies in different parts of the world, mostly in high-risk countries. A common protocol and questionnaire were used, and HPV DNA testing was done in two central research laboratories using the MYO9/11 and the General Primer (GP) GP5+/6+ PCR testing systems. Figure 7 shows the summary results on the HPV DNA prevalence in case and control specimens, the risk estimates and their confidence intervals for SCC. The figure shows very high Odds Ratios (ORs) with estimates in the range of 50 to 150 with several estimates in the several hundred range. These risk estimates lead to calculations of attributable fractions in the range of 90 to $95 \%$ [27].

The results are strikingly consistent in the literature for pre-invasive lesions, for SCC and adenocarcinomas and for studies that tested for HPV DNA as a group or studies that restricted the analyses to HR HPV types. Studies that have compared risk factors for CIN 3 and invasive cancer have not reported any significant differences in their associations with HPV or with their epidemiological profile (reviewed in [19]).

The pool of IARC studies was large enough to provide type-specific risk estimates for 18 types. Restricting the analyses to the studies that used the GP5+/6+ HPV detection system and to SCCs, the adjusted OR for HPV DNA detection (the factor by which the reference risk of cervical cancer is multiplied if HPV DNA is detected) was OR $=158.2$ (95\% Confidence Interval $(C I)=113.2-220.6)$. The risk estimates for adenocarcinomas was OR $=81.3$ (95\% CI $=42.0-157.1)$ [23]. Type specific risk estimates for squamous cell cancer are shown in Fig. 8 and were as follows: HPV 16: $\mathrm{OR}=435$; HPV 18: OR = 248; HPV 45: OR = 198; 
Table 2

Prevalence of the most common HPV types in cervical cancer by region

\begin{tabular}{|c|c|c|c|c|c|c|c|c|c|}
\hline \multicolumn{2}{|c|}{$\begin{array}{l}\text { Sub-Saharan } \\
\text { Africa }\end{array}$} & \multicolumn{2}{|c|}{$\begin{array}{l}\text { Northern } \\
\text { Africa }\end{array}$} & \multicolumn{2}{|c|}{$\begin{array}{l}\text { Central-South } \\
\text { America }\end{array}$} & \multicolumn{2}{|c|}{ South Asia } & \multicolumn{2}{|c|}{$\begin{array}{c}\text { Europe \& } \\
\text { North America }\end{array}$} \\
\hline HPV type & $\%$ & HPV type & $\%$ & HPV type & $\%$ & HPV type & $\%$ & HPV type & $\%$ \\
\hline HPV 16 & 47.7 & HPV 16 & 67.6 & HPV 16 & 57.0 & HPV 16 & 52.5 & HPV 16 & 69.7 \\
\hline HPV 18 & 19.1 & HPV 18 & 17.0 & HPV 18 & 12.6 & HPV 18 & 25.7 & HPV 18 & 14.6 \\
\hline HPV 45 & 15.0 & HPV 45 & 5.6 & HPV 31 & 7.4 & HPV 45 & 7.9 & HPV 45 & 9.0 \\
\hline HPV 33 & 3.2 & HPV 33 & 4.0 & HPV 45 & 6.8 & HPV 52 & 3.1 & HPV 31 & 4.5 \\
\hline HPV 58 & 3.2 & HPV 31 & 3.4 & HPV 33 & 4.3 & HPV 58 & 3.0 & HPV 56 & 2.2 \\
\hline
\end{tabular}

HPV: Human Papillomavirus.

Source of data: [Adapted from reference: N. Muñoz et al. [26].

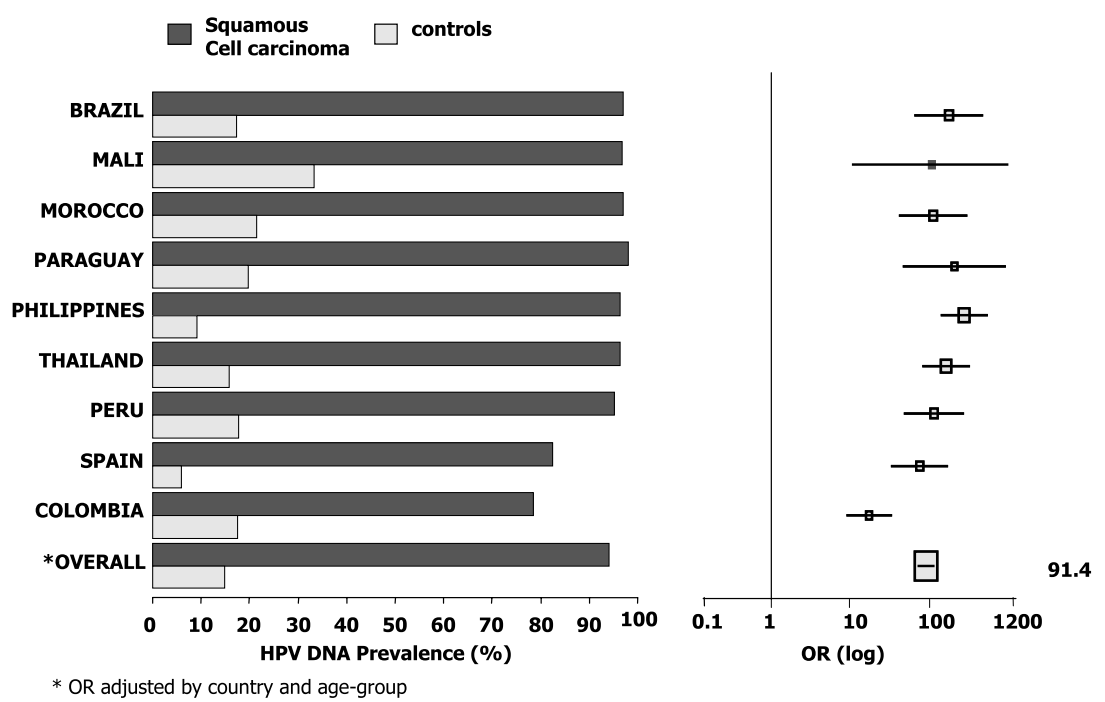

Source: Adapted from reference Muñoz N, et al., 2003 [27]

Fig. 7. HPV-DNA prevalence and odds ratios for squamous carcinoma of cervix by country.

HPV 31: OR = 124; HPV 52: OR = 200; HPV 33: $\mathrm{OR}=374 ;$ HPV 58: OR = 115; HPV 35: OR = 74; HPV 59: OR = 419; HPV 51: OR = 67; HPV 56: $\mathrm{OR}=45$; HPV 39: infinity; HPV 68: OR $=54$. The risk for any given high-risk type was not statistically different from the risk reported for HPV 16. Likewise, the proportion of multiple types in a given specimen varies across studies and particularly in relation to the HPV detection method used. However, in all studies of invasive carcinoma the risk linked to multiple HPV types does not vary significantly from the risk linked to single HPV types.

These studies and a recent International review done at IARC concluded that the evidence is now sufficient to consider as high-risk carcinogenic types HPVs 16 , $18,31,33,35,39,45,51,52,56,58,59,68,73$ and 82 [IARC, in press]. A second group of HPV types are rarely found in cases and have been classified as Lowrisk types and these include HPVs 6, 11, 40, 42, 43,
44, 54, 61, 70, 72, 81 and CP6108. From IARC's and other studies, a small group of HPV types remains in the category of uncertain risk currently including HPVs 26, 53, 66 and perhaps others.

\subsection{Cohort studies}

Repeated sampling of women being followed for viral persistence and cervical abnormalities has shown that the median duration of the infections is around 8 months for high risk HPV types as compared to 4.8 months for the low-risk HPV types. In unrelated studies, the time estimates were fairly consistent. In one study in a high-risk population in Brazil, the mean duration of HPV detection was 13.5 months for high-risk HPV types and 8.2 months for the non-oncogenic types. HPV 16 tended to persist longer than the average for high-risk types other than HPV 16. The results were remarkably similar in a student population in the Unit- 


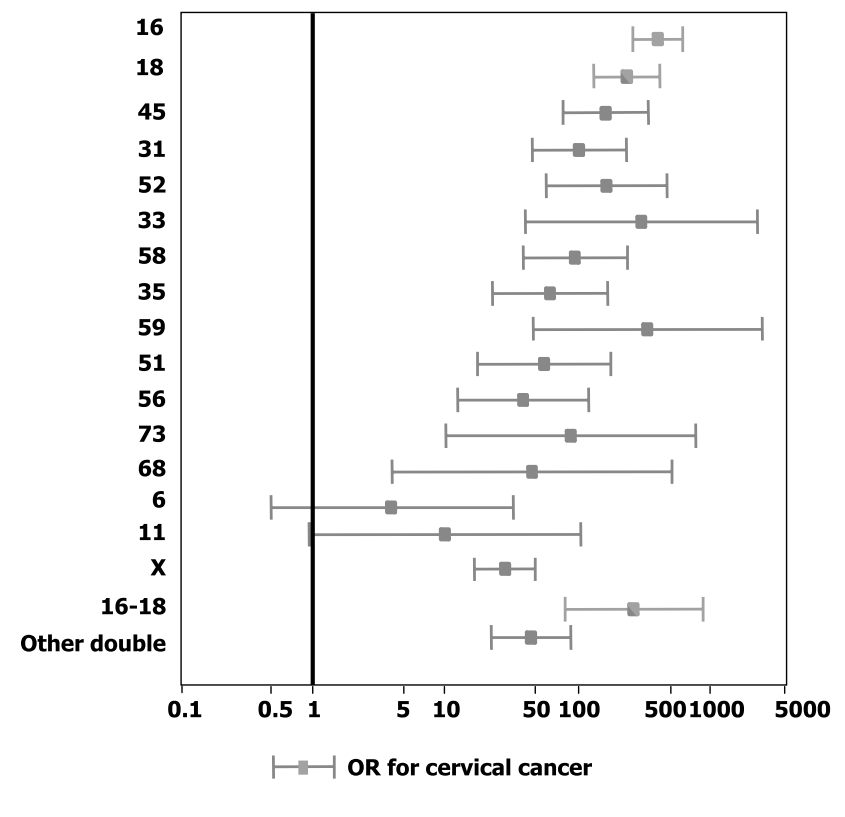

Fig. 8. HPV type-specific risk estimate for cervical cancer.

ed States and in the United Kingdom. However, the currently observed time intervals may still suffer from imprecision in the estimates of time at first exposure, from the variability in the end-point definition and from censoring due to treatment of the early lesions.

Follow-up studies of women with and without cervical abnormalities have indicated that the continuous presence of high risk HPV is necessary for the development, maintenance and progression to CIN 3 and above. A substantial fraction (i.e. 15-30\%) of women with high risk HPV DNA who are cytomorphologically normal at recruitment will develop CIN 2/3 within the subsequent 4-year interval. Conversely, among women found to be high risk HPV DNA negative and cytologically identified as atypical squamous cells of undetermined significance (ASCUS), or borderline or mild dysplasia, CIN 2/3 is unlikely to develop during a follow-up of two years and their cytology is likely to return to normal. Women found positive for-low risk HPVs rarely become persistent carriers and their probability of progression to CIN 2/3 is extremely low (reviewed in [19]).

As on going cohorts expand their follow up time, more precise estimates are being provided on the predictive value of viral persistence as defined by repeated measurements of viral types and variants. One of such cohorts in Sao Paulo has shown that the incidence of cervical lesions in women who were twice HPV-negative was 0.73 per 1,000 women-months. The corresponding incidence among women with repeated HPV-16 or HPV-18 positive results was 8.68 , a 12fold increased incidence. The OR for HPV persistence among women who were twice HPV positive for the same oncogenic types was $\mathrm{OR}=41.2(95 \% \mathrm{CI}=10.7-$ 158.3).

The follow up studies in Costa Rica are beginning to assess the potential for progression-to-neoplasic lesions of some of the most frequent high-risk HPV types. The study confirms that the risk of progression (given persistence) is significantly higher for HPV 16 and 18 HPV carriers over the risk related to any of the remaining high-risk types included in the cocktail test $\mathrm{HC} 2$ [4].

Finally, persistence of HPV DNA detection after treatment for CIN 2/3 is an accurate predictor of relapse, significantly more sensitive than repeated vaginal cytology within the 24 months following surgery [28]. These results are useful in defining the clinical role of HPV testing. In screening, in the management of ASCUS and the follow up of women following treatment for HSIL.

\section{Other environmental risk factors for cervical cancer}

Most of the sexual behaviour parameters that were linked to cervical cancer in the past are being re- 
evaluated in studies that considered the strong influence of the presence of HPV. Soon after the introduction of HPV testing in research protocols, it became clear that the key risk factors that reflected sexual behaviour, such as the number of sexual partners, merely reflected the probability of HPV exposure. Because of the growing evidence that HPV is a necessary factor in cervical cancer, it soon became a standard procedure in the reports of case control studies to include analyses restricted to HPV positive cases and controls to properly assess the contribution of additional factors to the risk of cervical cancer. In relation to invasive cervical cancer, the IARC's pooled HPV positive restricted analyses included HPV positive 1,768 cases and 262 HPV positive controls and the key findings concerning environmental risk factors are discussed briefly.

\subsection{Long term use of oral contraceptives}

Ever use of OC was associated with a significant increase in risk of cervical cancer $(\mathrm{OR}=1.47$ (95\% CI $=1.02-2.12$ ) with a clear dose response relationship with duration. The use of OCs for less than 5 years was not related to the risk of cervical cancer $(\mathrm{OR}=0.77$ $(95 \% \mathrm{CI}=0.46-1.29))$ but it increased significantly for 5-9 years of use $(\mathrm{OR}=2.72(95 \% \mathrm{CI}=1.36-$ $5.46)$ ) and for $10+$ years of use $(\mathrm{OR}=4.48(95 \% \mathrm{CI}$ $=2.24-9.36)$ ).

The evidence for an association of cervical cancer with the use of oral or other hormonal contraceptives is not entirely consistent. A number of studies that investigated HPV positive women found no associations or only weak associations with HSIL/CIN 3 in subgroup analyses. These apparently conflicting results may reflect the increased cytological surveillance of women that are taking OCs in developed countries and the use of different case definition (ASCUS up to HSIL/CIN3 as opposed to cervical cancer) in cohort studies.

Because of the potential public health importance of an interaction between long-term use of OCs and HPV infections in the development of cervical cancer, efforts are now being devoted to verify the results in different populations. A meta-analyses on the association between hormonal contraceptives and cervical cancer concludes that there is a linear dose response relationship and that the effect tends to return to average within a time interval of 5 to 10 years after OC cessation. The relevant units at the World Health Organization examined the evidence with an international working group that recognized the significance of the association. However, the group also concluded that, in balance, the benefits currently achieved by the use of OCs in developing countries (i.e. unwanted pregnancy avoidance) outbalance the increase in risk and should not require a major change in the current family planning strategies.

\subsection{High parity}

In the IARC case control studies, HPV positive women who reported seven or more full term pregnancies had a four-fold increased risk of cervical cancer as compared to similar HPV positive women that were nulliparous $(\mathrm{OR}=3.8(95 \% \mathrm{CI}=2.7-5.5))$. There was still a two-fold increased risk when women reporting 7 or more pregnancies were compared to HPV positive women who reported 1-2 full term pregnancies [29]. Similar results were obtained in Costa Rica and Thailand as well as among women with pre-invasive disease in the Portland Cohort Study. In Denmark and in the Manchester cohort study, two populations with low parity, the effects are less visible for pre-neoplastic lesions (reviewed in [30]).

It has been speculated that the general reduction in the average number of births in developed countries over the last decades may have contributed to the reduction in cervical cancer incidence but formal proof of the hypothesis has not yet been produced.

The combined effect in the risk of cervical cancer of parity and exposure to OCs has been examined and shown that at each level of OCs use there is an increased risk with a significant trend with the number of lifetime pregnancies. The trend is more remarkable as women refer use of OCs for extended periods of time. As compared to nulliparous women not having used OCs, use for longer that five years and having had more than five full term pregnancies increases the risk by a significant 11-fold [29]. In many of the studies reported, it was difficult to further analyse the association by type of OC use or by estrogens/progesterone dose levels. It is now recognized that current formulations have significantly reduced the product composition and the observed results might apply differently to current users of OCs. However, these observations underline the need to continue conducting studies on the interaction of steroids with the biology of HPV.

\subsection{Cigarette smoking}

The pooled results of the IARC's studies found that "ever smoking" was associated with a two-fold, statistically significant, increased risk of cervical cancer with 
a significant dose response. These findings are consistent with those found for "current vs. never smoking" among HPV positive women for pre-neoplastic cervical lesions in virtually all cohort studies in Costa $\mathrm{Ri}-$ ca $(\mathrm{OR}=2.3)$, the US Portland $(\mathrm{OR}=2.7$ for $\mathrm{CIN}$ $2-3)$, in Copenhagen $(\mathrm{OR}=1.9)$ and a large cohort study in Manchester $(\mathrm{OR}=2.2)$ [30]. These studies are providing growing evidence on the carcinogenic effect of cigarette smoking in women with persistent HPV infection. The monograph program at IARC reviewed the evidence in 2002 and concluded that smoking was an independent risk factor for cervical cancer [31]. However the mechanisms by which cigarette smoking may affect cervical cancer (i.e. a direct effect of the tobacco metabolites, an indirect effect related to tobacco-induced immunosupression or to reduced dietary antioxidants) remain elusive.

\subsection{Co-infection with the Human Immunodeficiency Virus}

The evidence of a putative interaction between HPV and Human Immunodeficiency Virus (HIV) in the origin of cervical cancer was formally recognized when cervical cancer was included as one of the criteria of acquired immune deficiency syndrome (AIDS) among HIV positive women. The subsequent literature largely confirmed the evidence although some major confounders of the epidemiological association tend to obscure the results notably the powerful impact of screening in some populations, the medical surveillance of HIV carriers in developed countries and the short survival time of HIV/AIDS patients in many populations at high risk of cervical cancer in relation to the time intervals between HPV infection and cervical cancer [32].

\subsection{Co-infection with other sexually transmitted infections}

Markers of exposure to two other Sexually Transmitted Infections (STIs) have been repeatedly although inconsistentcy found associated to cervical cancer. Results from the IARC's multicenter study found a 2-fold increased risk in cervical cancer for the presence of antibodies to $\mathrm{CT}(\mathrm{OR}=2.1(95 \% \mathrm{CI}=1.1-4.0))$ and of antibodies to HSV 2 (OR $=2.19(95 \% \mathrm{CI}=1.41-$ $3.40)[33,34]$. Non-specific inflammatory changes have also been related to modest increases in risk for preneoplastic cervical lesions among HPV positive women. The difficulties with the evaluations of such factors lie in the strong co-linearity observed among all STIs and the limitations of some of the biomarkers currently used to assess ever exposure or persistent exposure. to assess ever exposure or persistent exposure.

\section{Conclusion}

In the last two decades, the aetiology of cervical cancer has become a coherent description that includes the identification of a limited group of HPV types as the necessary, etiologic agents with a few additional co factors intervening as such in the presence of the viral DNA. The association is universal and the HPV type variability is geographically limited. The clinical implications of these findings have resulted in novel screening and vaccination strategies for the prevention of cervical cancer. Current vaccines may prompt a change in the paradigm of cervical cancer prevention.

\section{Acknowledgments}

We acknowledge Meritxell Nomen and Cristina Rajo who were responsible for the secretarial workload. Partial support has been received from the Fondo de Investigaciones Sanitarias, Spain (FIS PI030240 and FIS 01/1237), from the European Commission (QLG4CT-2000-01238 \& QLG4-CT-2001-30142), from the Agència de Gestió d'Ajuts Universitaris I de Recerca (2005SGR00695) and from the Instituto de Salud Carlos III (Red de CÁNCER RCESP C03/09 \& Red de Salut Pública RTICCC C03/10).

Partial support has been received from the Marato de TV3 Foundation (051530).

\section{References}

[1] T. Iftner and L.L. Villa, Chapter 12: Human papillomavirus technologies, J Natl Cancer Inst Monogr (2003), 80-88.

[2] G.M. Clifford, S. Gallus, R. Herrero, N. Munoz, P.J. Snijders, S. Vaccarella, P.T. Anh, C. Ferreccio, N.T. Hieu, E. Matos, M. Molano, R. Rajkumar, G. Ronco, S. de Sanjose, H.R. Shin, S. Sukvirach, J.O. Thomas, S. Tunsakul, C.J. Meijer and S. Franceschi, Worldwide distribution of human papillomavirus types in cytologically normal women in the International Agency for Research on Cancer HPV prevalence surveys: a pooled analysis, Lancet 366 (2005), 991-998.

[3] N. Munoz, F. Mendez, H. Posso, M. Molano, A.J. van Den Brule, M. Ronderos, C. Meijer and A. Munoz, Incidence, duration, and determinants of cervical human papillomavirus infection in a cohort of Colombian women with normal cytological results, J Infect Dis 190 (2004), 2077-2087.

[4] M.J. Khan, P.E. Castle, A.T. Lorincz, S. Wacholder, M. Sherman, D.R. Scott, B.B. Rush, A.G. Glass and M. Schiffman, The elevated 10-year risk of cervical precancer and cancer in women with human papillomavirus (HPV) type 16 or 18 and the possible utility of type-specific HPV testing in clinical practice, J Natl Cancer Inst 97 (2005), 1072-1079. 
[5] S.K. Kjaer, B. Chackerian, A.J.C. van der Brule, E.I. Svare, G. Paull, J.M.M. Walboomers, J.T. Schiller, J.E. Bock, M.E. Sherman, D.R. Lowy and C.J.L.M. Meijer, High-Risk Human Papillomavirus Is Sexually Transmitted: Evidence from a Follow-up Study of Virgins Starting Sexual Activity (Intercourse), Cancer Epidemiol Biomark Prev 10 (2001), 101-106.

[6] X. Castellsague, A. Ghaffari, R.W. Daniel, F.X. Bosch, N. Munoz and K.V. Shah, Prevalence of penile human papillomavirus DNA in husbands of women with and without cervical neoplasia: a study in Spain and Colombia, J Infect Dis $\mathbf{1 7 6}$ (1997), 353-361.

[7] F.X. Bosch, X. Castellsagué, N. Muñoz, S. de Sanjosé, A.M. Ghaffari, L.C. González, M. Gili, I. Izarzugaza, P. Viladiu, C. Navarro, A. Vergara, N. Ascunce, E. Guerrero and K.V. Shah, Male sexual behavior and Human Papillomavirus DNA: key risk factors for cervical cancer in Spain, J Natl Cancer Inst 88(15) (1996), 1060-1067.

[8] N. Muñoz, X. Castellsagué, F.X. Bosch, L. Tafur, S. de Sanjosé, N. Aristizabal, A.M. Ghaffari and K.V. Shah, Difficulty in Elucidating the Male Role in Cervical Cancer in Colombia, a High-Risk Area for the Disease, $J$ Natl Cancer Inst $\mathbf{8 8}(15)$ (1996), 1068-1075.

[9] S. de Sanjose, F.X. Bosch, I. Valls, M.P. Canadas, X. Castellsague, B. Lloveras and K.V. Shah, Prevalence of HPV cervical infections among imprisoned women in Barcelona, Spain, Sex Transm Infect 76 (2000), 58.

[10] S.K. Kjaer, E.I. Svare, A.M. Worm, J.M. Walboomers, C.J. Meijer and A.J. van Den Brule, Human papillomavirus infection in Danish female sex workers. Decreasing prevalence with age despite continuously high sexual activity, Sex Transm Dis 27 (2000), 438-445.

[11] X. Castellsague, F.X. Bosch, N. Munoz, C.J. Meijer, K.V. Shah, S. de Sanjose, J. Eluf-Neto, C.A. Ngelangel, S. Chichareon, J.S. Smith, R. Herrero, V. Moreno and S. Franceschi, Male circumcision, penile human papillomavirus infection, and cervical cancer in female partners, $N$ Engl $J$ Med 346 (2002), 1105-1112.

[12] J. Smith, R. Herrero, K. Erles, D. Grimm, N. Muñoz, F.X. Bosch, L. Tafur, K.V. Shah and J.R. Schlehofer, Adenoassociated virus seroposivility and HPV-induced cervical cancer in Spain and Colombia, Int J Cancer 94 (2001), 520-526.

[13] M.C. Bleeker, C.J. Hogewoning, F.J. Voorhorst, A.J. van Den Brule, P.J. Snijders, T.M. Starink, J. Berkhof and C.J. Meijer, Condom use promotes regression of human papillomavirusassociated penile lesions in male sexual partners of women with cervical intraepithelial neoplasia, Int J Cancer 107 (2003), 804-810.

[14] S. Franceschi, X. Castellsague, L. dal Maso, J.S. Smith, M. Plummer, C. Ngelangel, S. Chichareon, J. Eluf-Neto, K.V. Shah, P.J. Snijders, C.J. Meijer, F.X. Bosch and N. Munoz, Prevalence and determinants of human papillomavirus genital infection in men, Br J Cancer 86 (2002), 705-711.

[15] X. Castellsague, R.W. Peeling, S. Franceschi, S. de Sanjose, J.S. Smith, G. Albero, M. Diaz, R. Herrero, N. Munoz and F.X. Bosch, Chlamydia trachomatis infection in female partners of circumcised and uncircumcised adult men, Am J Epidemiol 162 (2005), 907-916.

[16] C. Munk, E.I. Svare, P. Poll, J.E. Bock and S. Krüger Kjaer, History of Genital Warts in 10,838 Women 20 to 29 Years of Age From the General Population, Sex Transm Dis 24(10) (1997), 567-572.

[17] C.J. Lacey, Therapy for genital human papillomavirus-related disease, J Clin Virol 32(Suppl 1) (2005), S82-S90.
[18] F.X. Bosch and T. Iftner, The Aetiology of Cervical Cancer Sheffield, UK: NHS Cervical Screening Programme, 2005.

[19] International Agency for Research on Cancer. IARC Handbooks of Cancer Prevention. Cervix Cancer Screening. Lyon: IARC Press, 2005.

[20] F.X. Bosch, A. Lorincz, N. Muñoz, C.J.L.M. Meijer and K.V. Shah, The causal relation between human papillomavirus and cervical cancer, J Clin Pathol 55 (2002), 244-265.

[21] F.X. Bosch, M.M. Manos, N. Munoz, M. Sherman, A.M. Jansen, J. Peto, M.H. Schiffman, V. Moreno, R. Kurman and K.V. Shah, Prevalence of human papillomavirus in cervical cancer: a worldwide perspective. International biological study on cervical cancer (IBSCC) Study Group, J Natl Cancer Inst 87 (1995), 796-802.

[22] J.M. Walboomers, M.V. Jacobs, M.M. Manos, F.X. Bosch, J.A. Kummer, K.V. Shah, P.J. Snijders, J. Peto, C.J. Meijer and N. Munoz, Human papillomavirus is a necessary cause of invasive cervical cancer worldwide, J Pathol 189 (1999), $12-19$.

[23] X. Castellsague, M. Diaz, S. de Sanjose, N. Munoz, R. Herrero, S. Franceschi, R.W. Peeling, R. Ashley, J.S. Smith, P.J. Snijders, C.J. Meijer and F.X. Bosch, Worldwide human papillomavirus etiology of cervical adenocarcinoma and its cofactors: implications for screening and prevention, J Natl Cancer Inst 98 (2006), 303-315.

[24] H.D. Strickler, J.M. Palefsky, K.V. Shah, K. Anastos, R.S. Klein, H. Minkoff, A. Duerr, L.S. Massad, D.D. Celentano, C. Hall, M. Fazzari, S. Cu-Uvin, M. Bacon, P. Schuman, A.M. Levine, A.J. Durante, S. Gange, S. Melnick and R.D. Burk, Human papillomavirus type 16 and immune status in human immunodeficiency virus-seropositive women, $J$ Natl Cancer Inst 95 (2003), 1062-1071.

[25] G.M. Clifford, J.S. Smith, M. Plummer, N. Muñoz and S. Franceschi, Human papillomavirus types in invasive cervical cancer worldwide: a meta-analysis, Br J Cancer 88 (2003), 63-73.

[26] N. Munoz, F.X. Bosch, X. Castellsague, M. Diaz, S. de Sanjose, D. Hammouda, K.V. Shah and C.J. Meijer, Against which human papillomavirus types shall we vaccinate and screen? The international perspective, Int J Cancer 111 (2004), 278285.

[27] N. Munoz, F.X. Bosch, S. de Sanjose, R. Herrero, X. Castellsague, K.V. Shah, P.J. Snijders and C.J. Meijer, Epidemiologic classification of human papillomavirus types associated with cervical cancer, $N$ Engl J Med 348 (2003), 518-527.

[28] M. Kyrgiou, G. Koliopoulos, P. Martin-Hirsch, M. Arbyn, W. Prendiville and E. Paraskevaidis, Obstetric outcomes after conservative treatment for intraepithelial or early invasive cervical lesions: systematic review and meta-analysis, Lancet 367 (2006), 489-498.

[29] N. Munoz, S. Franceschi, C. Bosetti, V. Moreno, R. Herrero, J.S. Smith, K.V. Shah, C.J. Meijer and F.X. Bosch, Role of parity and human papillomavirus in cervical cancer: the IARC multicentric case-control study, Lancet 359 (2002), 1093 1101.

[30] X. Castellsague and N. Munoz, Chapter 3: Cofactors in human papillomavirus carcinogenesis - role of parity, oral contraceptives, and tobacco smoking, J Natl Cancer Inst Monogr (2003), 20-28.

[31] IARC. IARC Monographs. Vol. 38. Tobacco Smoking and Tobacco Smoke. 2002.

[32] J.M. Palefsky and E.A. Holly, Chapter 6: Immunosuppression and co-infection with HIV, J Natl Cancer Inst Monogr (2003), 41-46. 
[33] J.S. Smith, R. Herrero, C. Bosetti, N. Munoz, F.X. Bosch, J. Eluf-Neto, X. Castellsague, C.J. Meijer, A.J. van Den Brule, S. Franceschi and R. Ashley, Herpes simplex virus-2 as a human papillomavirus cofactor in the etiology of invasive cervical cancer, J Natl Cancer Inst 94 (2002), 1604-1613.
[34] J.S. Smith, N. Munoz, R. Herrero, J. Eluf-Neto, C. Ngelangel, S. Franceschi, F.X. Bosch, J.M. Walboomers and R.W. Peeling, Evidence for Chlamydia trachomatis as a human papillomavirus cofactor in the etiology of invasive cervical cancer in Brazil and the Philippines, J Infect Dis 185 (2002), 324-331. 


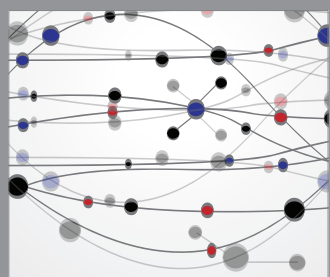

The Scientific World Journal
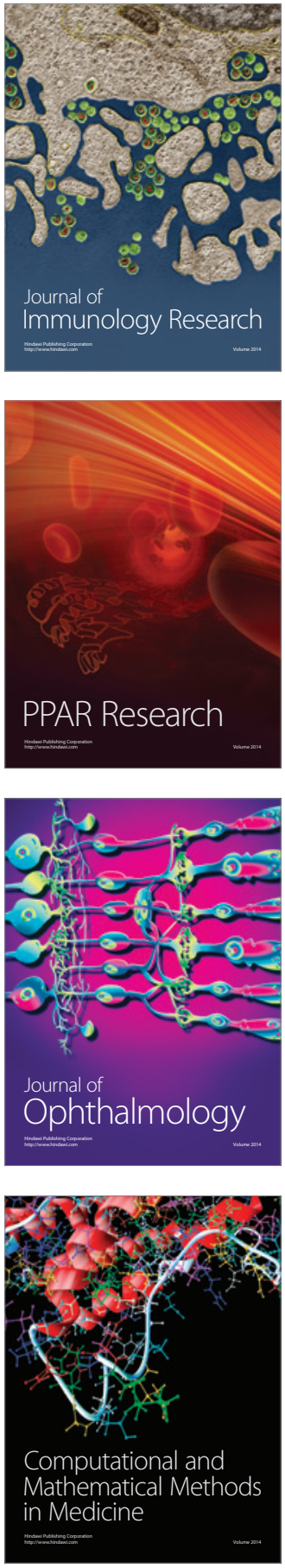

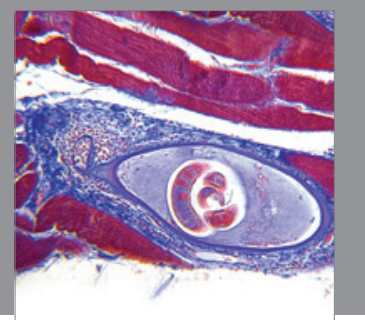

Gastroenterology

Research and Practice
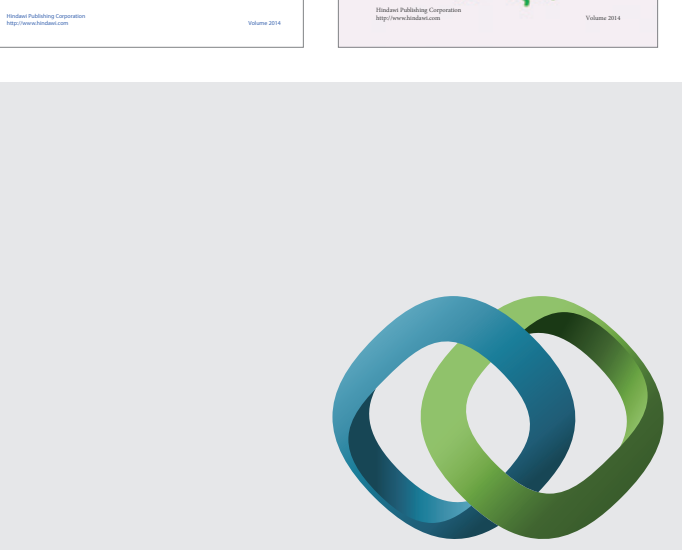

\section{Hindawi}

Submit your manuscripts at

http://www.hindawi.com
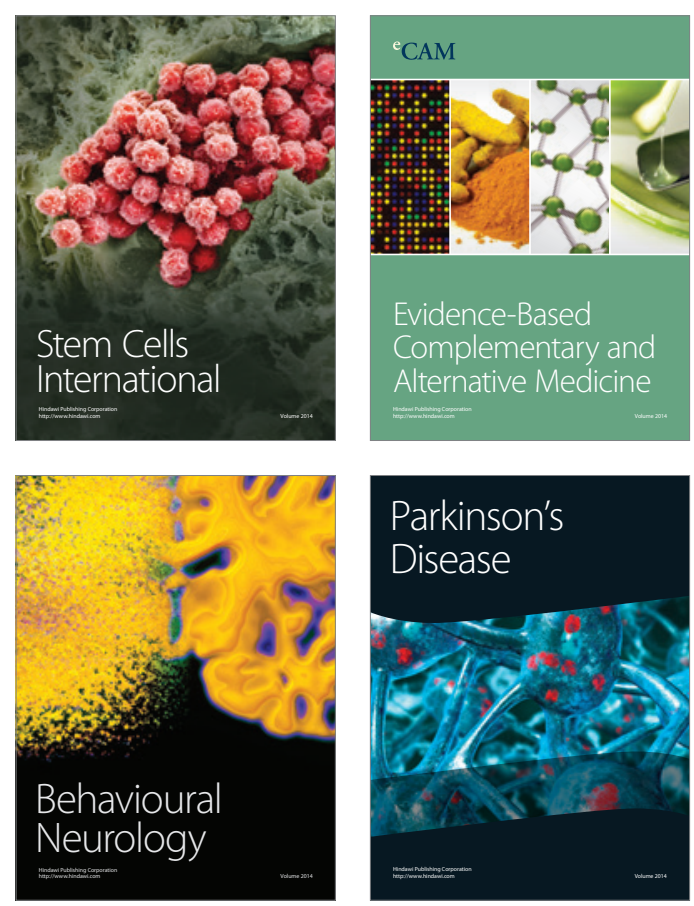

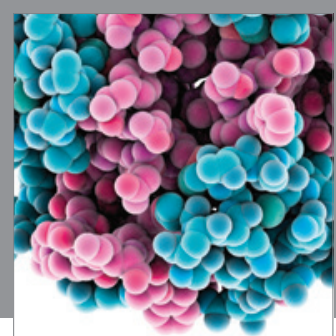

Journal of
Diabetes Research

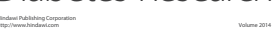

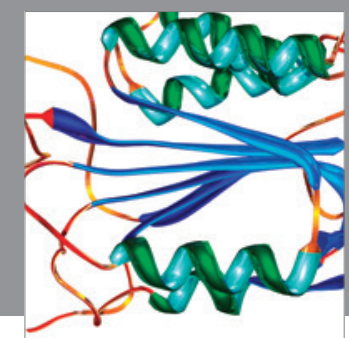

Disease Markers
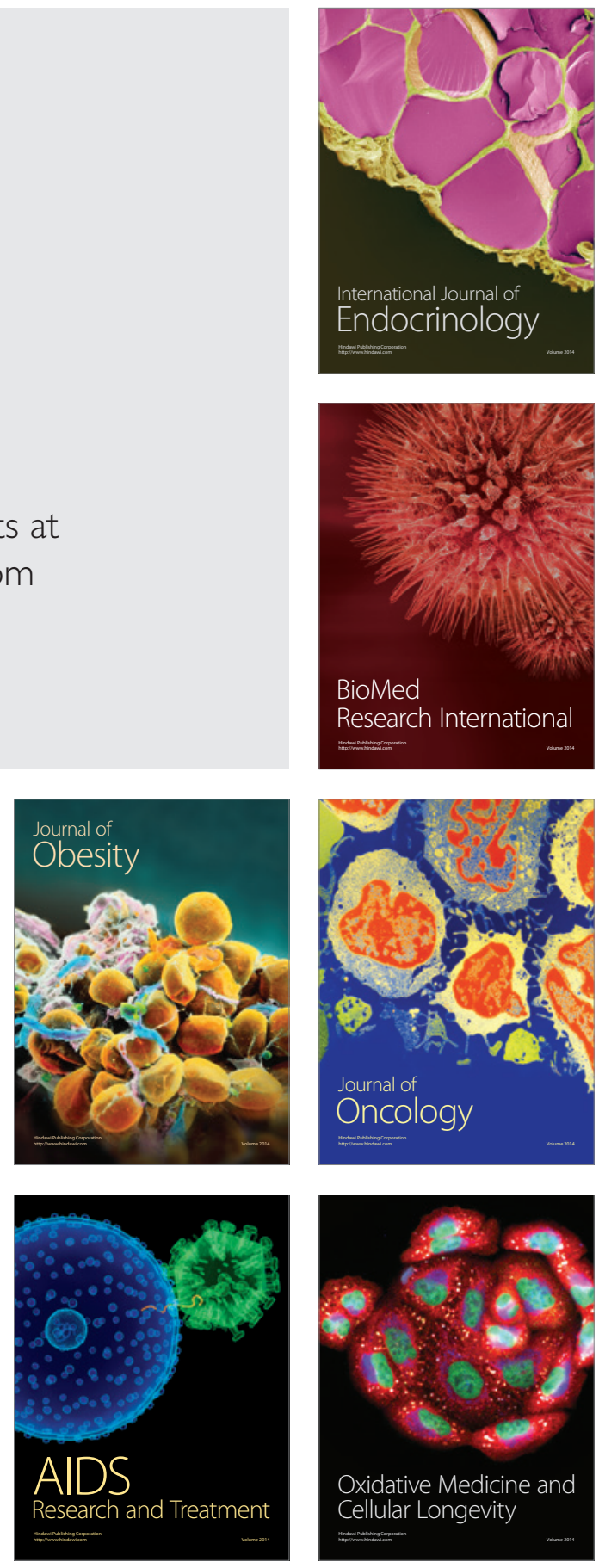